\title{
Perineal recurrence of prostate cancer six years after trans-perineal brachytherapy
}

\author{
Wietse Eppinga, MD', Peter Vijverberg, MD², Rien Moerland, MD, PhD', Eric Brand", Jochem van der Voort van Zyp, \\ MD, PhD', Juus Noteboom, MD, PhD', Marco van Vulpen, MD, PhDl \\ 'Department of Radiation Oncology, University Medical Center Utrecht, The Netherlands, ${ }^{2}$ Department of Urology, St. Antonius Hospital \\ Nieuwegein, The Netherlands
}

\begin{abstract} excision.

\section{Purpose}

After perineal needle biopsies of the prostate, seeding of cancer cells along the needle tract can occur in upto $1 \%$ of patients with prostate cancer [1]. Even more rarely, perineal seeding can occur after laparoscopic radical prostatectomy [2], cryosurgery [3] or radioactive seed brachytherapy [4]. The optimal management of perineal seeding of prostate cancer in absence of distant metastases is unknown, and currently, local excision is the most common approach [5]. We describe the second case in the literature, to our knowledge, of perineal seeding of prostate cancer cells after brachytherapy (iodine-125), and propose a novel, potentially-curative, minimally-invasive treatment option using stereotactic radiotherapy.
\end{abstract}

We report a case of perineal recurrence of prostate cancer 6 years after low-dose-rate (LDR) brachytherapy for localized prostate cancer. The most common approach to treat such perineal masses, including those occurring after prior biopsy or surgery, is local excision. We report the use of stereotactic radiotherapy with volumetric modulated arc therapy (VMAT) as a novel non-invasive, potentially curative, and patient-friendly alternative to local

Key words: brachytherapy, perineal seeding, prostate cancer, radiotherapy, seeds.

\section{Material and methods}

A 59-year old male was diagnosed with a Gleason $3+3$ $=6$ adenocarcinoma of the right peripheral zone of the prostate based on positive transrectal biopsies. The tumor was staged cT1cNxMx, and the initial prostate specific antygen (PSA) was $6.4 \mathrm{ng} / \mathrm{ml}$. Final staging was determined based on clinical examination, including digital rectal exam, and magnetic resonance imaging (MRI). The patient elected to undergo treatment with brachytherapy, delivered as low-dose-rate (LDR) treatment of the whole prostate. A total of $53^{125}$ I seeds were transperineally inserted into the prostate through 22 needles under ultrasound guidance. Intra-operative dosimetry confirmed an excellent plan: the dose to the hottest $90 \%$ of the pros- tate $\left(D_{90}\right)$ was $193 \mathrm{~Gy}$, and $99 \%$ of the prostate volume was covered by $100 \%$ of the prescribed dose of $145 \mathrm{~Gy}$. There were no complications during the procedure or abnormalities at post-implant dosimetry.

The patient recovered well from the procedure without relevant long-term toxicity. A PSA nadir of $0.11 \mathrm{ng} / \mathrm{ml}$ was reached 5 years afterwards. However, subsequently a gradual raise in PSA was noticed. At 6-years post-treatment, the PSA level was $4.3 \mathrm{ng} / \mathrm{ml}$, and the PSA-doubling time (PSA-DT) was 14 months. An ${ }^{11} \mathrm{C}$-choline positron emission tomography (PET)/computed tomography (CT) $\left({ }^{11} \mathrm{C}\right.$-choline PET/CT) followed by an MRI of the small pelvis revealed a perineal mass with a diameter of $14 \mathrm{~mm}$ just dorsally to the penile bulb (Fig. 1). Subsequent needle aspiration biopsy confirmed the diagnosis of recurrent prostate cancer. The position of the recurrence in the area of the brachytherapy needle tracts is most consistent with perineal seeding by one of the brachytherapy needles.

The patient was discussed at multidisciplinary tumor rounds. In light of the patient's young age, low co-morbidity index, low PSA-DT, and absence of other metastases on ${ }^{11} \mathrm{C}$-choline PET/CT, local treatment was recommended. Local excision was considered risky due to the close relation of the nodule with the penile bulb, the anal sphincter and the previously irradiated prostate, and therefore stereotactic radiotherapy was suggested.

\section{Results}

In order to optimally delineate the perineal recurrent tumor, the previously acquired ${ }^{11} \mathrm{C}$-choline PET/CT, MRI,
Address for correspondence: Wietse Eppinga, MD, Department of Radiation Oncology, UMC Utrecht, Heidelberglaan 100, 3584 CX Utrecht, The Netherlands, phone: +31 88755 8800, fax: +31 88755 5850, e-mail: w.s.c.eppinga@umcutrecht.nl
Received: 22.07.2014

Accepted: 27.10 .2014

Published: 30.12.2014 
and 3D ultrasound images were digitally matched to the radiotherapy planning $\mathrm{CT}$ dataset. The anal sphincter, rectal wall, urethra, and the previously treated prostate were considered to be "organs at risk" (OAR) in order to minimizes doses to these structures.

A volumetric modulated arc therapy (VMAT) plan was created. This type of therapy is a relatively new form of delivering radiation to a target volume by rotating the gantry of the linear accelerator around the patient with the radiation beam continuously on. While the gantry is rotating with the beam on, the gantry speed, beam shape, and output (dose rate) are modulated according to the optimized treatment plan. Using VMAT, the total treatment time can be significantly reduced, while even better coverage of the tumor or sparing of OAR can be achieved compared to more conventional radiotherapy techniques. The patient was treated to a dose of $35 \mathrm{~Gy}$ in 5 fractions to the tumor with acceptable low dose to surrounding OAR (Fig. 2). To confirm accurate setup, 2 gold markers were implanted into the perineal nodule under ultrasound guidance. These fiducials were used for daily online imaging to ensure accurate targeting. All 5 fractions were delivered within 2 weeks on outpatient basis. For each treatment session, the average time in the treatment room was 10 minutes, and beam-on time was approximately 3 minutes.

Patient-reported toxicity consisted of minor fatigue and a burning sensation of the anus for several weeks. All complaints were self-limiting. Three months after VMAT external beam treatment, the PSA returned to $<0.1 \mathrm{ng} /$ $\mathrm{ml}$. One year after treatment PSA remained undetectable. No chronic toxicity was reported by the patient.

\section{Discussion}

We describe a rare case of a patient who developed a perineal prostate cancer seeding metastasis after LDR brachytherapy for localized prostate cancer. Since the original diagnosis of the prostate cancer was based on transrectal biopsies, and no other procedures breached the area around the penile bulb, the seeding must have been caused by one of the brachytherapy needles during the procedure.

This is the first case of perineal seeding occurring in our hospital, where over 2400 patients have been treated

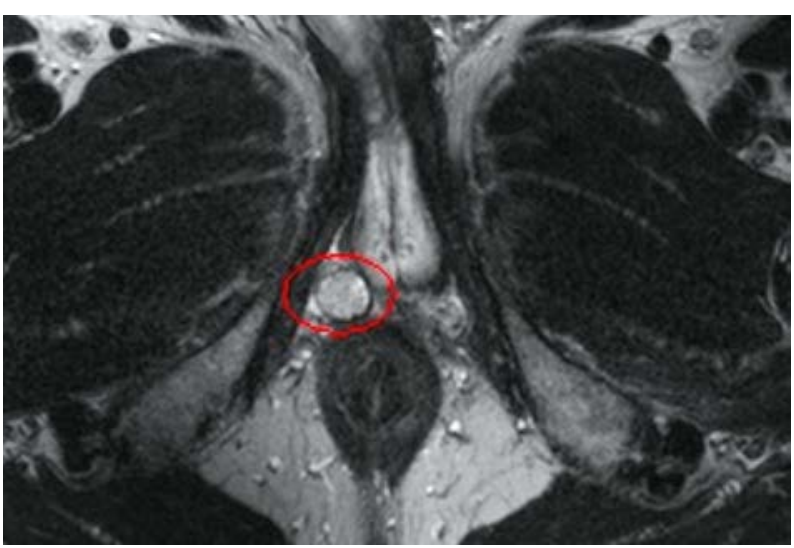

Fig. 1. T2-weighted image showing perineal metastasis between penile bulb and sphincter with transperineal LDR brachytherapy for prostate cancer since 1989. In a review of the literature, we could only identify one similar case of seeding after brachytherapy, as reported by Teh et al. [4]. However, this risk has been recognized previously: Downey et al. [3] suggested that "perineal seeding might occur more frequently in the future due to an expected increase in modern minimally invasive surgical treatments that develop a transperineal tract". Another possible risk factor for perineal seeding may be the increased use of MR guided biopsies in recent years. In addition, ascertainment of such recurrences may be easier with modern imaging technologies, such as the increased use of ${ }^{11} \mathrm{C}$-PET/CT in patients with biochemical recurrences [6], which could otherwise be underreported. In Figure 2 it is clear that the reported perineal recurrence was located in the proximity of the previously treated prostate. Merrick et al. [7] reported in a series of 4547 patients a significant underdosage in the first $5 \mathrm{~mm}$ outside the prostate in patients treated with brachytherapy. They describe a $V_{100}$ (the volume of prostate receiving the prescription dose) as low as $45 \%$ in this $5 \mathrm{~mm}$ annulus. The brachytherapy dose outside the prostate for this patient was clearly too low to eradicate all seeded tumor cells.

The prognostic value of perineal seeding is unclear. Moul et al. [1] describes a cohort of 502 patients who had pathology-proven prostate cancer and perineal biopsies. Five patients $(1 \%)$ were found to have perineal seeding. They also evaluated 14 more published cases of seeding. These patients were detected before the routine availability of CT, MRI, PET, and PSA testing. In this small group, distant metastases were discovered within 16 months for all patients. None of them lived longer than 3 years after diagnosis. The authors reported that the greatest risk factors for developing perineal seeding seem to be biopsy of

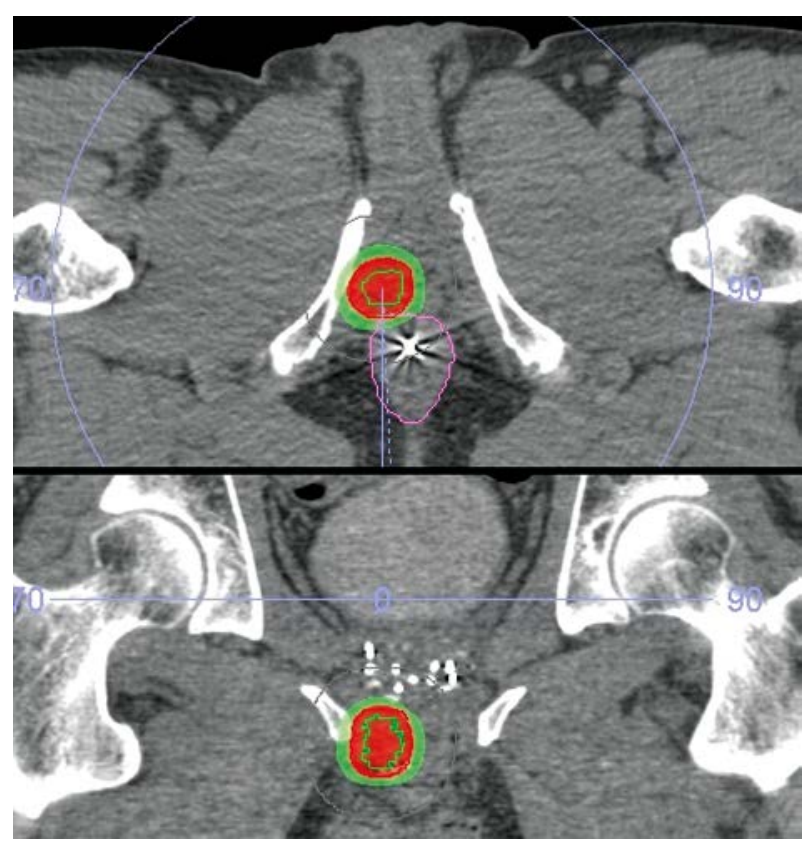

Fig. 2. Axial and coronal view of perineal metastasis (red line), prescribed dose of $35 \mathrm{~Gy}$ (red color wash) and $25 \mathrm{~Gy}$ (green color wash). Anal canal (purple) with lead string for demarcation 
a large volume local tumor, and the technique of biopsy. However, other authors report better results. A review by Haddad et al. [5] shows considerable better prognosis with $60 \%$ of patients being alive and well 18 -months after treatment. We hypothesize that survival may be considerably better in selected patients who initially were presented with low risk prostate cancer, when diagnosed earlier or recur with low PSA-DTs. Such patients may benefit from salvage local treatments if they are younger without significant co-morbidities.

The interval between perineal biopsies or transperineal treatment and seeding varies greatly. In this case, the interval was 7 years between diagnosis and salvage treatment. An interval between several months [5] and 14 years has been described [8], the latter possibly related to more favorable immunohistochemical characteristics of the tumor.

Due to recent advances in medical imaging and radiotherapy techniques, it is now possible to detect small metastases earlier and irradiate them with high doses in a limited number of fractions. The stereotactic VMAT technique we described in this case is ideal for small nodular masses like perineal seeding metastases. The delivered dose can be considered lethal for prostate cancer cells as has been shown by Loblaw et al. [9]. The limited number of 5 fractions compared to 30-40 conventionally fractioned treatments for prostate cancer and the limited toxicity of the treatment are patient-friendly. However, such treatment would be expected to have some toxicity risks, depending on the dose in the surrounding OARs, including risks of fecal soiling, anal pain or ulceration, and urethral stricture. With a dose of lower than $25 \mathrm{~Gy}$ in 5 fractions to the OARs, this risk is very limited (Fig. 2).

Since surgical management of perineal seeded prostate cancer metastases is potentially toxic and is more invasive, we suggest stereotactic radiotherapy treatment as a promising non-invasive alternative. Due to the uncommon nature of these recurrences, management by a multidisciplinary team is strongly recommended.

\section{Conclusions}

A rare case of perineal seeding due to a LDR brachytherapy procedure for localized prostate cancer is presented. In the era of PSA testing and improved imaging, perineal locoregional recurrences might be detected more frequently. Stereotactic radiotherapy can be a feasible, patient-friendly, potentially curative alternative to local excision in selected patients.

\section{Disclosure}

Authors report no conflict of interest.

\section{References}

1. Moul JW, Miles BJ, Skoog SJ, McLeod DG. Risk factors for perineal seeding of prostate cancer after needle biopsy. J Urol 1989; 142: 86-88.

2. Shin YS, Doo AR, Kim MK et al. First case of peritoneal seeding of prostate cancer during robot-assisted laparoscopic radical prostatectomy. Can J Urol 2012; 19: 6303-6305.
3. Downey DB, Chin JL, Williams JC. Perineal prostate cancer seeding along needle tract following cryosurgery. Can J Urol 1999; 6: 823-825.

4. Teh BS, Chou CC, Schwartz MR et al. Perineal prostatic cancer seeding following radioactive seed brachytherapy. J Urol 2001; 166: 212 (Abstract).

5. Haddad FS, Somsin AA. Seeding and perineal implantation of prostatic cancer in the track of the biopsy needle: three case reports and a review of the literature. J Surg Oncol 1987; 35: 184-191.

6. Krause BJ, Souvatzoglou M, Tuncel M et al. The detection rate of [11C]Choline-PET/CT depends on the serum PSA-value in patients with biochemical recurrence of prostate cancer. Eur J Nucl Med Mol Imaging 2008; 35: 18-23.

7. Merrick GS, Butler WM, Grimm P et al. Permanent prostate brachytherapy extracapsular radiation dose distributions: analysis of a multi-institutional database. J Contemp Brachytherapy 2013; 5: 117-121.

8. Moul JW, Bauer JJ, Srivastava S et al. Perineal seeding of prostate cancer as the only evidence of clinical recurrence 14 years after needle biopsy and radical prostatectomy: molecular correlation. Urology 1998; 51: 158-160.

9. Loblaw A, Cheung P, D'Alimonte L et al. Prostate stereotactic ablative body radiotherapy using a standard linear accelerator: toxicity, biochemical, and pathological outcomes. Radiother Oncol 2013; 107: 153-158. 